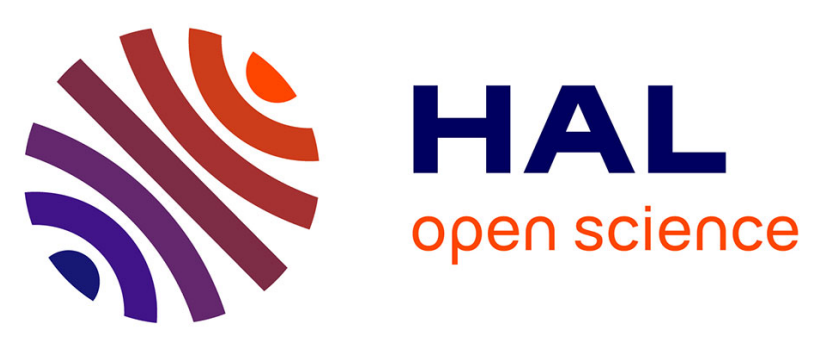

\title{
A brief note on the computation of the Bödewadt flow with Navier slip boundary conditions
}

Bikash Sahoo, Saied Abbasbandy, Sébastien Poncet

\section{To cite this version:}

Bikash Sahoo, Saied Abbasbandy, Sébastien Poncet. A brief note on the computation of the Bödewadt flow with Navier slip boundary conditions. Computers and Fluids, 2014, 90, pp.133-137. 10.1016/j.compfluid.2013.11.020 . hal-00976676

\section{HAL Id: hal-00976676 https://hal.science/hal-00976676}

Submitted on 10 Apr 2014

HAL is a multi-disciplinary open access archive for the deposit and dissemination of scientific research documents, whether they are published or not. The documents may come from teaching and research institutions in France or abroad, or from public or private research centers.
L'archive ouverte pluridisciplinaire HAL, est destinée au dépôt et à la diffusion de documents scientifiques de niveau recherche, publiés ou non, émanant des établissements d'enseignement et de recherche français ou étrangers, des laboratoires publics ou privés. 


\title{
A brief note on the computation of the Bödewadt flow with Navier slip boundary conditions
}

\author{
Bikash Sahoo $^{1}$ Saeid Abbasbandy ${ }^{2}$ Sébastien Poncet $^{3} *$ \\ ${ }^{1}$ Department of Mathematics, National Institute of Technology, Rourkela, Odisha, INDIA \\ Phone +9106612462706, E mail: bikashsahoo@nitrkl.ac.in \\ 2 Department of Mathematics, Science and Research Branch, Islamic Azad University, \\ Tehran, 14778, IRAN, E mail: abbasbandy@yahoo.com \\ 3 Aix-Marseille Université, CNRS, Ecole Centrale, Laboratoire M2P2 UMR 7340 , \\ Marseille, FRANCE \\ E mail: sebastien.poncet@univ-amu.fr; Tel. 0033(0)491118555 \\ * Corresponding author
}

\begin{abstract}
In this short communication, numerical solutions are obtained for the steady Bödewadt flow of a viscous fluid subject to partial slip boundary conditions. The resulting system of nonlinear and fully coupled similarity equations are integrated accurately by a finite difference scheme and by the Keller-box method. It is observed that slip has a prominent effect on the velocity field, reducing drastically the axial velocity and the pressure. Moreover, the torque required to maintain the disk at rest decreases for increasing values of slip.
\end{abstract}

Keywords: Rotating flow, Partial slip, Finite difference method, Keller-box method

MSC codes here, in the form:

2000 MSC: 76U05, 76A05, 34B15

\section{Introduction}

The steady laminar flow of a viscous incompressible fluid near a rotating disk, originally solved by von Kármán [1], is one of the few problems in fluid dynamics for which the Navier-Stokes equations admit an exact solution. The twin problem arising when the fluid rotates with a uniform angular velocity at a large distance from a stationary disk, is known as the Bödewadt flow. This problem, for a viscous incompressible fluid, also admits an exact solution for the Navier-Stokes equations, subject to the conventional no-slip boundary conditions, as shown theoretically by Bödewadt [2]. The flow is characterized by the radial pressure gradient being balanced by the centrifugal forces. Fluid flows towards the axis of rotation and swepts upwards. The boundary layer, which develops on the disk, produces a secondary flow of stagnation type in the von Kármán case and of wake type in the Bödewadt problem. Batchelor [3] suggested 
that for large Reynolds numbers, the rotor-stator flow consists of boundary layers on each disk separated by a core of fluid rotating as a solid body. It can be seen as the connection of a Von Kármán flow along the rotor with a Bödewadt flow along the stator. Nowadays, this type of flow still receives a constant attention by the introduction of more complex and combined phenomena: heat transfer, non-Newtonian fluid [5, 6], magnetic field or partial slip.

All the studies mentioned above admit no-slip condition on the walls, which is more a hypothesis than a condition deduced from any principle. Evidence of the fluid slip on a solid surface has been reported by Matthews and Hill [7]. For example, if one considers a zero-thickness disk admitting a stress-free condition on its surface and rotating around its axis, it does not modify the motion of the surrounding fluid, which would remain at rest. It confirms an intuitive result that the boundary condition on the disk plays a key role on the fluid motion. Slip condition has also some industrial relevance when the fluid is composed of emulsions, suspensions, foams or polymer solutions. In other situations where the wall surface is rough, the no-slip boundary condition also becomes impractical to apply exactly. The proper boundary condition is then well described by the general Navier's condition [8], where the amount of relative slip is proportional to the local shear stress through the slip length(es). If the characteristic scale of roughness is small compared to the boundary layer thickness, the no-slip condition may be well approximated by a partial slip condition [8]. Miklavčič and Wang [9] have considered the von Kármán swirling flow of a viscous fluid with slip boundary condition. More recently, Sherwood [10] solved the flow due to a zero-thickness disk rotating around its axis by the use of Hankel transforms. The combined effects of slip and non-Newtonian cross-viscous parameter on the rotating flows past free rotating disks have been thoroughly studied by Sahoo [11] and Sahoo and Poncet [12].

A literature survey shows that no particular attention has been paid to the effects of slip on the Bödewadt flow of a viscous fluid. The present work is devoted to study the effects of slip on the steady Bödewadt flow of a viscous fluid. A second order finite difference method and an effective Keller box method are used to solve the fully coupled and highly nonlinear differential equations. The present paper is a step forward in the computation of the rotor-stator flow with partial slip effects to establish reference solutions for numerical benchmarks.

\section{Formulation of the problem}

One considers a viscous fluid occupying the space $z>0$ over an infinite stationary disk, which coincides with $z=0$. The motion is due to the rotation of the fluid like a rigid body with constant rotation rate $\Omega$ at a large distance from the disk. One shall assume that the principal directions of the roughness are the radial and azimuthal, i.e. a concentrically grooved disk [9], but the results could also apply to the case of a randomly rough disk. The flow is described in the cylindrical polar coordinates $(r, \phi, z)$ with the rotational symmetry, $\frac{\partial}{\partial \phi} \equiv 0$. Let $\mathbf{V}=(u, v, w)$ be the fluid velocity vector. Considering the usual boundary 
layer approximations and using the similarity transform [1]:

$u=r \Omega F(\zeta), \quad v=r \Omega G(\zeta), \quad w=\sqrt{\Omega \nu} H(\zeta), \quad z=\sqrt{\frac{\nu}{\Omega}} \zeta, \quad p-p_{\infty}=-\rho \nu \Omega P$

the equations of continuity and motion take the form $[2,4]$ :

$$
\begin{aligned}
& \frac{d H}{d \zeta}+2 F=0, \\
& \frac{d^{2} F}{d \zeta^{2}}-H \frac{d F}{d \zeta}-F^{2}+G^{2}=1, \\
& \frac{d^{2} G}{d \zeta^{2}}-H \frac{d G}{d \zeta}-2 F G=0 \\
& \frac{d P}{d \zeta}-H \frac{d H}{d \zeta}+\frac{d^{2} H}{d \zeta^{2}}=0
\end{aligned}
$$

The no-slip boundary conditions in terms of similarity variables become,

$$
\begin{aligned}
& \zeta=0: \quad F=0, \quad G=0, \quad H=0, \\
& \zeta \rightarrow \infty: \quad F \rightarrow 0, \quad G \rightarrow 1, \quad P \rightarrow 0 .
\end{aligned}
$$

A generalization of the Navier's partial slip condition $[8,9]$ gives, in the radial and azimuthal directions:

$$
\begin{aligned}
& \left.u\right|_{z=0}=\left.\lambda_{1} \bar{\tau}_{r z}\right|_{z=0} \\
& \left.v\right|_{z=0}=\left.\lambda_{2} \bar{\tau}_{\phi z}\right|_{z=0}
\end{aligned}
$$

where $\lambda_{1}, \lambda_{2}$ are the slip coefficients, and $\bar{\tau}_{r z}, \bar{\tau}_{\phi z}$ are the physical components of the stress tensor. One defines the dimensionless slip coefficients as:

$$
\lambda=\lambda_{1} \sqrt{\frac{\Omega}{\nu}} \mu, \quad \eta=\lambda_{2} \sqrt{\frac{\Omega}{\nu}} \mu .
$$

With the help of the transformations (1), the corresponding partial slip boundary conditions (7)-(8) become:

$$
\begin{array}{r}
F(0)=\lambda F^{\prime}(0), \quad G(0)=\eta G^{\prime}(0), \quad H(0)=0, \\
F(\infty) \rightarrow 0, \quad G(\infty) \rightarrow 1, \quad P(\infty) \rightarrow 0 .
\end{array}
$$

\section{Finite difference solution}

The finite difference method (FDM) has been used to solve the system of coupled nonlinear Eqs. (2)-(5) subject to the slip boundary conditions (10). The semi-infinite domain $[0, \infty)$ is replaced by a finite domain $\left[0, \zeta_{\infty}\right)$, with $\zeta_{\infty}$ sufficiently large so that the numerical solution closely approximates the terminal boundary conditions. One approximates the functions and their derivatives by their finite difference counterparts to solve a sequence of linear systems. 
1. One solves:

$$
F^{\prime \prime}-H^{(k)} F^{\prime}=\left(F^{(k)}\right)^{2}-\left(G^{(k)}\right)^{2}+1
$$

using the derivative boundary conditions (10) and denotes the solution of $(11)$ as $\tilde{F}^{(k+1)}$. To obtain convergence, one defines $F^{(k+1)}$ by the following smoothing formula:

$$
F^{(k+1)}=\alpha_{1} \tilde{F}^{(k+1)}+\left(1-\alpha_{1}\right) \tilde{F}^{(k)}, \quad 0 \leq \alpha_{1} \leq 1
$$

2. The same procedure is successively used for the $G$ and $H$ components and then for the pressure $P$ :

$$
\begin{aligned}
& G^{\prime \prime}-H^{(k)} G^{\prime}=2 F^{(k+1)} G^{(k)} \\
& G^{(k+1)}=\alpha_{2} \tilde{G}^{(k+1)}+\left(1-\alpha_{2}\right) \tilde{G}^{(k)}, \quad 0 \leq \alpha_{2} \leq 1 \\
& H^{\prime}=-2 F^{(k+1)} \\
& H^{(k+1)}=\alpha_{3} \tilde{H}^{(k+1)}+\left(1-\alpha_{3}\right) \tilde{H}^{(k)}, \quad 0 \leq \alpha_{3} \leq 1 \\
& P^{\prime}-2 F^{\prime}=-2 H^{(k+1)} F^{(k+1)} \\
& P^{(k+1)}=\alpha_{4} \tilde{P}^{(k+1)}+\left(1-\alpha_{4}\right) \tilde{P}^{(k)}, \quad 0 \leq \alpha_{4} \leq 1
\end{aligned}
$$

3. The iterations start with suitable initial guesses $F^{(0)}, G^{(0)}$ and $H^{(0)}$, borrowed from the work by Sahoo and Poncet [12]. If $\left(F^{(k+1)}, F^{(k)}\right),\left(G^{(k+1)}, G^{(k)}\right)$, $\left(H^{(k+1)}, H^{(k)}\right)$ and $\left(P^{(k+1)}, P^{(k)}\right)$ are close enough to each other, one stops, otherwise one sets $k=k+1$ and goes back to step 1 .

In order to solve the above system of equations by finite difference method, we introduce a grid in $0 \leq \zeta \leq \zeta_{\infty}$ by dividing it into $n$ equal parts with a mesh size $h$ equal to 0.01 . It has been verified that this value guarantees a grid independent solution. One approximates the derivatives by:

$$
F^{\prime}\left(\zeta_{i}\right)=\frac{F_{i+1}-F_{i-1}}{2 h}, \quad F^{\prime \prime}\left(\zeta_{i}\right)=\frac{F_{i+1}-2 F_{i}+F_{i-1}}{h^{2}}, \quad i=1,2, \ldots n-1
$$

In order to obtain a diagonally dominant linear algebraic system for Eqs. (11) and (13), $F^{\prime}$ and $G^{\prime}$ are discretized by backward difference approximations as $H_{i}^{(k+1)}>0$ for Bödewadt flow. One obtains:

$$
\begin{gathered}
{\left[1+h H_{i}^{(k)}\right] F_{i-1}+\left[-2-h H_{i}^{(k)}\right] F_{i}+F_{i+1}=h^{2}\left[\left(F^{(k)}\right)^{2}-\left(G^{(k)}\right)^{2}+1\right]} \\
{\left[1+h H_{i}^{(k)}\right] G_{i-1}+\left[-2-h H_{i}^{(k)}\right] G_{i}+G_{i+1}=2 h^{2} F_{i}^{(k+1)} G_{i}^{k}}
\end{gathered}
$$

Finally, Eqs. (15) and (17) are discretized by central difference approximations. The above algebraic system is solved by a generalized Gauss-Seidel method instead of a successive over relaxation method. The convergence of the generalized Gauss-Seidel method for the above diagonally dominant system is reached after 15 iterations to achieve an accuracy of $O\left(10^{-6}\right)$. The FORTRAN 90 code was compiled and run using the NIT Rourkela server composed of Dual Intel Xeon (8 Gb RAM, 4 Gbps Lan card). 


\section{Solution by Keller-box method}

The nonlinear Eqs. (3)-(4), by considering (2), are reduced to:

$$
\begin{aligned}
& \frac{d^{3} H}{d \zeta^{3}}-H \frac{d^{2} H}{d \zeta^{2}}+\frac{1}{2}\left(\frac{d H}{d \zeta}\right)^{2}-2 G^{2}+2=0 \\
& \frac{d^{2} G}{d \zeta^{2}}-H \frac{d G}{d \zeta}+G \frac{d H}{d \zeta}=0
\end{aligned}
$$

and boundary conditions (10) are converted to:

$H^{\prime}(0)=\lambda H^{\prime \prime}(0), \quad G(0)=\eta G^{\prime}(0), \quad H(0)=0, \quad H^{\prime}(\infty) \rightarrow 0, \quad G(\infty) \rightarrow 1 .(24)$

New dependent variables $S(\zeta), Q(\zeta)$ and $T(\zeta)$ are introduced such that the Eqs. (22)-(23) can be written as:

$$
\begin{aligned}
& \frac{d H}{d \zeta}=S(\zeta) \\
& \frac{d S}{d \zeta}=Q(\zeta), \\
& \frac{d G}{d \zeta}=T(\zeta) \\
& \frac{d Q}{d \zeta}-H Q+\frac{1}{2} S^{2}-2 G^{2}+2=0, \\
& \frac{d T}{d \zeta}-H T+S G=0 .
\end{aligned}
$$

The boundary conditions (24) become:

$$
S(0)=\lambda Q(0), \quad G(0)=\eta T(0), \quad H(0)=0, \quad S(\infty) \rightarrow 0, \quad G(\infty) \rightarrow 1 .
$$

One considers the segment $\left[\zeta_{j-1}, \zeta_{j}\right]$, with the midpoint $\zeta_{j-1 / 2}$ :

$$
\zeta_{0}=0, \zeta_{j}=\zeta_{j-1}+h, \zeta_{J}=\zeta_{\infty},
$$

where $h=0.01$ is the $\Delta \zeta$-spacing and $j=1,2, \cdots, J$ is a sequence number that indicates the coordinate location. The finite difference approximations of Eq. (25)-(29) are written at the midpoint $\zeta_{j-1 / 2}$, as: 


$$
\begin{aligned}
& H_{j}-H_{j-1}-\frac{1}{2} h\left(S_{j}+S_{j+1}\right)=0, \\
& S_{j}-S_{j-1}-\frac{1}{2} h\left(Q_{j}+Q_{j+1}\right)=0, \\
& G_{j}-G_{j-1}-\frac{1}{2} h\left(T_{j}+T_{j+1}\right)=0, \\
& Q_{j}-Q_{j-1}-\frac{1}{4} h\left(H_{j}+H_{j-1}\right)\left(Q_{j}+Q_{j-1}\right)+\frac{1}{8} h\left(S_{j}+S_{j-1}\right)^{2}- \\
& \quad \frac{1}{2} h\left(G_{j}+G_{j-1}\right)^{2}+2 h=0, \\
& T_{j}-T_{j-1}-\frac{1}{4} h\left(H_{j}+H_{j-1}\right)\left(T_{j}+T_{j-1}\right)+\frac{1}{4} h\left(S_{j}+S_{j-1}\right)\left(G_{j}+G_{j-1}\right) .
\end{aligned}
$$

The Eqs.(32)-(36) are imposed for $j=1,2, \cdots, J$ for sufficiently large $\zeta_{\infty}$. The boundary conditions (30) are converted to:

$$
S_{0}=\lambda Q_{0}, \quad G_{0}=\eta T_{0}, \quad H_{0}=0, \quad S_{J}=0, \quad G_{J}=1 .
$$

One can linearize the nonlinear system (32)-(36) by the Newton's method, and the linearized system, which has a block-tridiagonal structure, can then be solved by the block elimination method. The MATHEMATICA code for the Keller-box method was run on a 64-bit Windows machine with Intel Core i7 and $3.4 \mathrm{GHz}$ processor.

\section{Results and discussions}

To validate the two numerical approaches, the values of $F, G$ and $H$ for the no-slip case $(\lambda=\eta=0)$ are compared to those reported by Owen and Rogers [4] in Table 1. An excellent agreement is thus obtained with relative differences of the extrema far less than $0.01 \%$.

In order to have an insight of the effects of slip on the Bödewadt flow, results are shown for the uniform roughness $(\lambda=\eta)$ using the finite difference method only. More comparisons between the two approaches are provided at the end of this section. It is noteworthy that, for other combinations of $\lambda$ and $\eta$ (not shown here), the behavior remains the same. Figs. 1a to 1c show the effects of slip on the velocity field. Fig. 1a shows the variation of the radial velocity component $F$ with the slip parameter $\lambda$. The effect of slip on $F$ is prominent near the disk. Clearly, the maximum value of the radial inflow decreases with an increase in slip and its location moves towards the disk. The radial velocity gets positive away from the disk and approaches the asymptotic value at a shorter distance from the disk for larger $\lambda$. Multiple cross-overs are observed for the radial velocity profiles. Fig. $1 \mathrm{~b}$ shows the azimuthal velocity $G(\zeta)$. Its value increases with $\zeta$, reaches its maximum, then decreases and reaches its asymptotic value $G=1$. One can observe that $G$ increases with an increase in slip, 
near the disk and decreases away from the disk, as expected. The oscillations occurring in the boundary layer when the fluid rotates near a stationary disk can be explained in the following manner. The radial inflow, induced by a low tangential velocity in the vicinity of the stationary disk, tends to conserve the angular momentum and thus tends to increase the tangential velocity with decreasing radius. For an overshoot, radial advection of the angular momentum near the disk must be strong enough to do more than balancing the dissipation of angular momentum caused by the wall shear. This inward radial advection of surplus angular momentum is possible as long as the distribution of circulation in the outer flow increases with increasing radius. A local overshoot in the tangential velocity increases the centrifugal force locally which then tends to induce a radial outflow. This radial outflow convects an angular momentum defect to force an undershoot in the tangential velocity, and the above process is repeated to yield oscillatory approach to infinity. The variation of the axial velocity component $H$ with $\lambda$ can be seen in Fig. 1c. It is observed that $H$ decreases significantly with an increase in slip. The axial profiles become flatter with an increase in $\lambda$. The axial velocity at infinity, $H_{\infty}$ is strongly reduced compared to the no-slip case $\lambda=0$, for which $H_{\infty}=1.349421$. It is interesting to find that slip dominates oscillations in the velocity profiles.

The prominent effect of slip on the pressure distribution can be observed in Fig. 1d. It is evident that in absence of slip, for $\lambda(=\eta)=0$, the dimensionless pressure $P$ has a dominating effect near the stationary disk. This induces a radial inward flow near the disk surface. Pressure decreases with an increase in slip near the disk and consequently, the strength of the radial inflow decreases, as shown in Fig. 1a. In presence of slip, the pressure profiles attain their asymptotic value $P=0$ closer to the disk surface.

The tangential $\bar{\tau}_{\phi}$ and radial $\bar{\tau}_{r}$ shear stresses and the dimensionless moment coefficient $C_{m}$ are defined as:

$$
\tau_{\phi}=\frac{\bar{\tau}_{\phi}}{\rho r \sqrt{\nu \Omega^{3}}}=G^{\prime}(0), \quad \tau_{r}=\frac{\bar{\tau}_{r}}{\rho r \sqrt{\nu \Omega^{3}}}=F^{\prime}(0), \quad C_{m}=\frac{-\pi G^{\prime}(0)}{\sqrt{\mathbb{R}}}
$$

where $\mathbb{R}=\frac{R^{2} \Omega}{\nu}$ is the Reynolds number based on the radius and the tip velocity. Throughout the computation, the value of $\mathbb{R}$ has been fixed as $\mathbb{R}=1$. This definition of $C_{m}$ is an extension of the finite disk problem, which supposes that the disk radius is large enough. In the following, these three quantities, denoted with $*$, are normalized by their value obtained for $\lambda=0$, such that $C_{m}^{*}(\lambda)=C_{m}(\lambda) / C_{m}(\lambda=0)$ for example.

Figure 2 depicts the effects of slip on the normalized surface shear stress in the radial direction $\tau_{r}^{*}$ and compares the finite difference and Keller box methods. It is noticeable that $\tau_{r}$ remains negative throughout the range of the slip parameter with $\tau_{r}(\lambda=0)=-0.941971$. The present results show that the magnitude of $\tau_{r}^{*}$ decreases with an increase in $\lambda$. It is also reduced by a factor 4 between $\lambda=0$ and $\lambda=1$. The tangential shear stress $\tau_{\phi}^{*}$ and the moment coefficient $C_{m}^{*}$ in their dimensionless form are strictly equal. Figure 2 shows that they both decrease with an increase in slip. $\tau_{\phi}$ remains always positive for 
the entire range of slip parameter with $\tau_{\phi}(\lambda=0)=0.772886$. On the contrary, $C_{m}$ exhibits negative values with $C_{m}(\lambda=0)=-2.428092$. It may be attributed to the flow problem. The Von Kármán flow considered by Sahoo [11] is precisely the inverse problem, which explains the different signs for $C_{m}$. It is important to note that the linear nature of the $\tau_{r}^{*}, \tau_{\phi}^{*}$ and $C_{m}^{*}$ profiles for the current viscous flow is contrary to corresponding profiles for non-Newtonian flows [11, 12]. This may be due to the presence of extra nonlinear terms in the expressions for $\tau_{r}, \tau_{\phi}$ and $C_{m}$, associated with the non-Newtonian cross-viscous parameter [11, 12]. For these three quantities, there is an excellent agreement between the finite difference and the Keller-box methods.

To compare the efficiency of the aforementioned two numerical schemes, Table 2 shows the CPU time (in seconds) for different values of the slip parameter and a given mesh distribution $\left(\zeta_{\infty}=14\right.$ and $\left.h=0.01\right)$. Though the FDM is more effective than the KBM in some sense, the CPU time for the FDM is significantly much higher as compared to the Keller-box method. It should be taken with certain caution as the methods have been run using different machines with different configurations. However, the calculations remain quite fast and for both schemes, the CPU time increases with an increase in $\lambda$.

\section{Conclusions}

In this brief note, an effective second order finite difference method and the Keller box method are adopted to solve the system of coupled, highly nonlinear differential equations, arising due to the Bödewadt flow of a viscous fluid, subject to partial slip boundary conditions. It is found that slip decreases the radial inflow near the disk. With an increase in slip, the azimuthal velocity $G$ increases near the disk and decreases away from the disk. It is also observed that slip dominates the oscillations in the velocity profiles. One of the significant findings of the present study is that the moment coefficient $C_{m}$, which is a measure of the torque required to maintain the disk at rest, decreases with an increase in slip.

[1] Th. Von Kármán. Über laminare und turbulente Reibung. Z. Angew. Math. Mech., 1:233-252, 1921.

[2] U.T. Bödewadt. Die Drehströmung über festem Grunde. Z. Angew. Math. Mech., 20:241-253, 1940.

[3] G.K. Batchelor. Note on the class of solutions of the Navier-Stokes equations representing staedy non-rotationally symmetric flow. Q.J. Mech. Appl. Math., 4:29-41, 1951.

[4] J.M. Owen and R.H. Rogers. Flow and heat transfer in rotating disk systems. Vol.1: Rotor-Stator systems. Research Stud. Press Ltd, John Wiley \& Sons, New-York, 1989.

[5] B. Sahoo. Steady Bödewadt flow of a non-Newtonian Reiner-Rivlin fluid. Diff. Eqn. Dyn. Syst., 20:367-376, 2012. 
[6] B. Sahoo, R.A. Van Gorder, and H.I. Andersson. Steady revolving flow and heat transfer of a non-Newtonian Reiner-Rivlin fluid. Int. Comm. Heat Mass Trans., 39:336-342, 2012.

[7] M.T. Matthews and J.M. Hill. Newtonian flow with nonlinear Navier boundary condition. Acta Mech., 191(3-4):195-217, 2007.

[8] C.L.M. Navier. Sur les lois du mouvement des fluides. Comp. Rend. Acad. Sci., 6:389-440, 1827.

[9] M. Miklavčič and C.Y. Wang. The flow due to a rough rotating disk. $Z$. Angew. Math. Phys., 55:235-246, 2004.

[10] J.D. Sherwood. Resistance coefficients for Stokes flow around a disk with a Navier slip condition. Phys. Fluids, 24:093103, 2012.

[11] B. Sahoo. Effects of partial slip, viscous dissipation, Joule heating on Von Kármán flow and heat transfer of an electrically conducting non-Newtonian fluid. Comm. Nonlin. Sci. Num. Sim., 14:2982-2998, 2009.

[12] B. Sahoo and S. Poncet. Effects of slip on steady Bödewadt flow of a non-Newtonian fluid. Comm. Nonlin. Sci. Num. Sim., 17:4181-4191, 2012. 


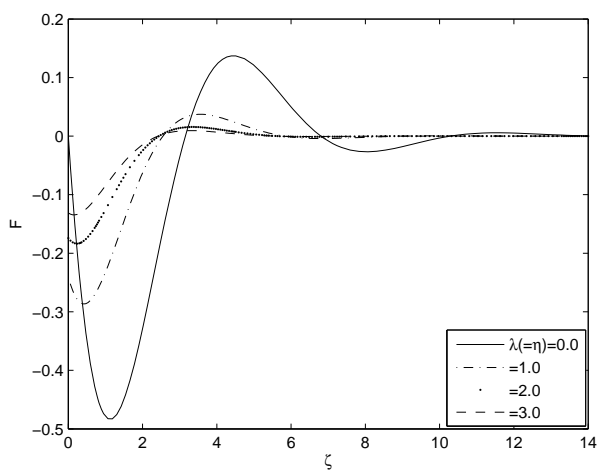

(a)

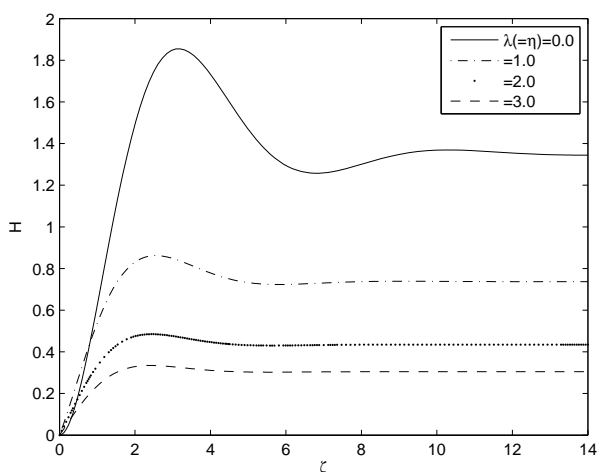

(c)

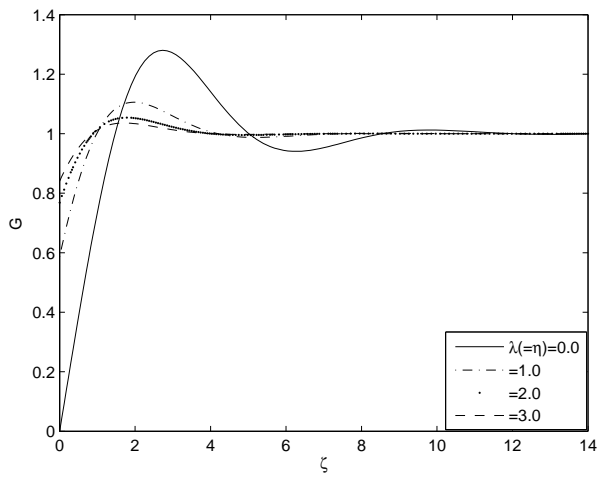

(b)

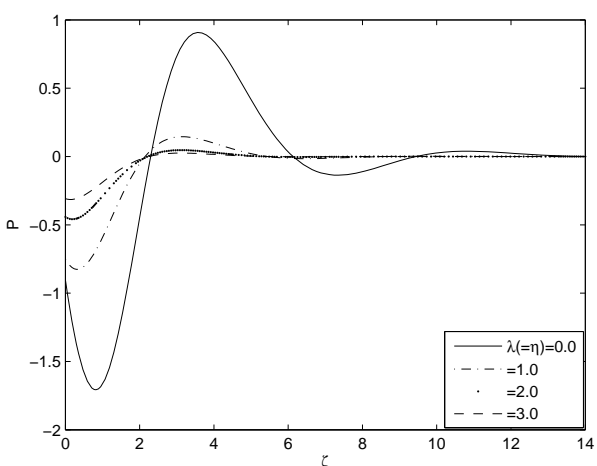

(d)

Figure 1: Variation with $\lambda(=\eta$ ) of (a) F, (b) G, (c) H and (d) P. Results obtained using the FDM. 


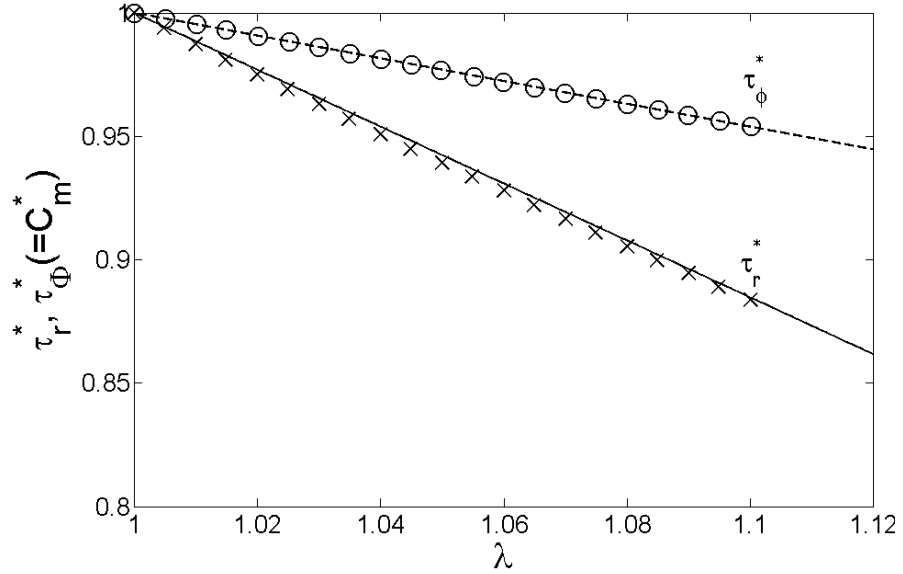

Figure 2: Variations of $\tau_{r}^{*}, \tau_{\phi}^{*}\left(=C_{m}^{*}\right)$ with $\lambda(=\eta)$. Comparisons between the FDM (lines) and the Keller box method (symbols). 
Table 1: Variations of $F, G$ and $H$ for $\lambda(=\eta)=0$.

\begin{tabular}{|c|c|c|c|c|c|c|c|c|c|}
\hline \multirow[b]{2}{*}{$\zeta$} & \multicolumn{3}{|c|}{$F$} & \multicolumn{3}{|c|}{$G$} & \multicolumn{3}{|c|}{${ }_{H}$} \\
\hline & FDM & Keller-Box & Owen \& Rogers [4] & FDM & Keller-Box & Owen \& Rogers [4] & FDM & Keller-Box & Owen \& Rogers [4] \\
\hline 0.0 & 0.000000 & 0.000000 & 0.0000 & 0.000000 & 0.000000 & 0.0000 & 0.000000 & 0.000000 & 0.0000 \\
\hline 0.5 & -0.348651 & -0.348651 & -0.3487 & 0.383430 & 0.383427 & 0.3834 & 0.194374 & 0.194365 & 0.1944 \\
\hline 1.0 & -0.478766 & -0.478769 & -0.4788 & 0.735429 & 0.735425 & 0.7354 & 0.624103 & 0.624090 & 0.6241 \\
\hline 1.5 & -0.449634 & -0.449639 & -0.4496 & 1.013401 & 1.013399 & 1.0134 & 1.098743 & 1.098730 & 1.0987 \\
\hline 2.0 & -0.328745 & -0.328751 & -0.3287 & 1.192367 & 1.192368 & 1.1924 & 1.492876 & 1.492870 & 1.4929 \\
\hline 2.5 & -0.176207 & -0.176210 & -0.1762 & 1.272136 & 1.272140 & 1.2721 & 1.745870 & 1.745870 & 1.7459 \\
\hline 3.0 & -0.036086 & -0.036088 & -0.0361 & 1.271405 & 1.271411 & 1.2714 & 1.849641 & 1.849640 & 1.8496 \\
\hline 3.5 & 0.066311 & 0.066310 & 0.0663 & 1.218219 & 1.218225 & 1.2182 & 1.830807 & 1.830810 & 1.8308 \\
\hline 9.5 & -0.010216 & -0.010217 & -0.0102 & 1.011849 & 1.011850 & 1.0118 & 1.361698 & 1.361690 & 1.3617 \\
\hline 10.0 & -0.003282 & -0.003283 & -0.0033 & 1.012120 & 1.012121 & 1.0121 & 1.368330 & 1.368320 & 1.3683 \\
\hline 10.5 & 0.001820 & 0.001819 & 0.0018 & 1.009906 & 1.009907 & 1.0099 & 1.368882 & 1.368880 & 1.3689 \\
\hline 11.0 & 0.004738 & 0.004738 & 0.0047 & 1.006537 & 1.006537 & 1.0065 & 1.365423 & 1.365420 & 1.3654 \\
\hline 11.5 & 0.005681 & 0.005681 & 0.0057 & 1.003090 & 1.003090 & 1.0031 & 1.360067 & 1.360060 & 1.3601 \\
\hline 12.0 & 0.005170 & 0.005171 & 0.0052 & 1.000271 & 1.000272 & 1.0003 & 1.354546 & 1.354540 & 1.3545 \\
\hline 12.5 & 0.003827 & 0.003827 & 0.0038 & 0.998411 & 0.998411 & 0.9984 & 1.350003 & 1.350000 & 1.3500 \\
\hline 20.0 & 0.000102 & 0.000102 & - & 0.999893 & 0.999893 & - & 1.349325 & 1.349320 & - \\
\hline 25.0 & 0.000009 & 0.000009 & - & 1.000014 & 1.000013 & . & 1.349457 & 1.349450 & . \\
\hline 25.5 & 0.000011 & 0.000011 & - & 1.000007 & 1.000007 & - & 1.349447 & 1.349440 & - \\
\hline 26.0 & 0.000010 & 0.000010 & - & 1.000001 & 1.000001 & . & 1.349437 & 1.349430 & . \\
\hline 26.5 & 0.000008 & 0.000008 & - & 0.999997 & 0.999997 & - & 1.349428 & 1.349420 & - \\
\hline 28.0 & 0.000000 & 0.000000 & 0.0000 & 1.000000 & 1.000000 & 1.0000 & 1.349421 & 1.349410 & 1.3494 \\
\hline
\end{tabular}


Table 2: Tabulation of CPU time (in seconds) for different values of $\lambda(=\eta)$ for 15 iterations.

\begin{tabular}{ccc}
\hline \hline$\lambda(=\eta)$ & FDM & Keller-box method \\
0 & 820.2 & 21.5 \\
1 & 852.6 & 21.7 \\
2 & 906.6 & 25.6 \\
3 & 931.8 & 25.7 \\
\hline \hline
\end{tabular}

